\title{
WEIGHTED INEQUALITIES FOR GEOMETRIC MEANS
}

\author{
B. OPIC AND P. GURKA
}

(Communicated by Andrew M. Bruckner)

\begin{abstract}
A characterization of weights $u, v$ is given for which the geometric mean operator $T f(x)=\exp \left(\frac{1}{x} \int_{0}^{x} \ln f(t) d t\right)$, defined for $f$ positive a.e. on $(0, \infty)$, is bounded from $L^{p}((0, \infty) ; v d x)$ to $L^{q}((0, \infty) ; u d x), p \in(0, \infty)$ and $q \in[p, \infty)$.
\end{abstract}

\section{INTRODUCTION}

(1.1)

We investigate the inequality

$$
\left(\int_{0}^{\infty}\left[\exp \left(\frac{1}{x} \int_{0}^{x} \ln f(t) d t\right)\right]^{q} u(x) d x\right)^{1 / q} \leq C\left(\int_{0}^{\infty} f^{p}(x) v(x) d x\right)^{1 / p}
$$

where $f$ is positive a.e. on $(0, \infty), u, v$ are weights, $p \in(0, \infty), q \in[p, \infty)$, and $C$ is a positive constant independent of $f$.

If $p=q=1$, then (1.1) is the limit case of the well-known Hardy inequality involving the averaging operator $\frac{1}{x} \int_{0}^{x} f(t) d t$, and its characterization was given by Heinig, Kerman, and Krbec (cf. [3]). If, in addition, $u(x) \equiv 1 \equiv v(x)$, then (1.1) holds with $C=e$ (this result is due to Knopp, see, e.g., [2, Theorem 335]).

One can show that an easy modification of ideas used in [3] provides the following result which generalizes that of [3] (for notation see below).

Theorem. Let $p \in(0, \infty), q \in[p, \infty)$, and let $u, v \in \mathscr{W}(0, \infty)$. Then there exists $C \in(0, \infty)$ such that the inequality (1.1) holds for all $f \in L_{+}^{p}((0, \infty) ; v)$ if and only if there is $\alpha>1$ such that

$$
B(\alpha, q, p):=\sup _{x>0} x^{(\alpha-1) / p}\left(\int_{x}^{\infty} \frac{u(t)}{t^{\alpha q / p}}\left[\exp \left(\frac{1}{t} \int_{0}^{t} \ln \frac{1}{v(\tau)} d \tau\right)\right]^{q / p} d t\right)^{1 / q}<\infty .
$$

Moreover, if $C$ is the least constant for which (1.1) holds, then

$$
\sup _{\alpha>1}\left(\frac{\alpha-1}{\alpha}\right)^{1 / p} B(\alpha, q, p) \leq C \leq \inf _{\alpha>1} e^{(\alpha-1) / p} B(\alpha, q, p) .
$$

Received by the editors March 30, 1992 and, in revised form, June 15, 1992.

1991 Mathematics Subject Classification. Primary 26D15, 26D10; Secondary 47H19. 
Let us note that some sufficient conditions for weighted inequalities (1.1) to hold with more general geometric means and with $p=q=1$ were found by Love [4]. His work is a generalization of the paper [1] by Cochran and Lee.

In this paper we suppose that $p \in(0, \infty), q \in[p, \infty)$ and we make use of "the method of differential equations" in order to obtain several types of conditions which are both necessary and sufficient for (1.1) to hold. In our method some ideas from [5-7,3] are employed. Let us point out that "the method of differential equations" is used here for the first time in the context of the geometric mean operator. The main result of the paper is formulated as Theorem 2.1.

Setting $g(t)=\ln f(t)$, the inequality (1.1) becomes

$$
\left(\int_{0}^{\infty}\left[\exp \left(\frac{1}{x} \int_{0}^{x} g(t) d t\right)\right]^{q} u(x) d x\right)^{1 / q} \leq C\left(\int_{0}^{\infty}[\exp g(x)]^{p} v(x) d x\right)^{1 / p},
$$

which is an exponential-type inequality involving the averaging operator $\frac{1}{x} \int_{0}^{x} g(t) d t$.

Throughout the paper we use the following

Notation. By $\mathscr{W}(0, \infty)$ we denote the set of all weights, i.e., the set of all functions measurable, positive, and finite almost everywhere on $(0, \infty)$. For $p \in(0, \infty)$ and $v \in \mathscr{W}(0, \infty)$ we define

$$
L_{+}^{p}((0, \infty) ; v):=\left\{f \in \mathscr{W}(0, \infty) ; \int_{0}^{\infty} f^{p}(x) v(x) d x<\infty\right\} .
$$

The symbol $A C((0, \infty))$ stands for the collection of all functions absolutely continuous on every compact subinterval $[a, b] \subset(0, \infty)$. Finally, we denote by $P(0, \infty)$ the set of all positive, finite, and measurable functions on $(0, \infty)$.

\section{THE METHOD OF DIFFERENTIAL EQUATIONS}

First, let us formulate the main result of the paper.

Theorem 2.1. Let $p \in(0, \infty), q \in[p, \infty), u, v \in \mathscr{W}(0, \infty)$, and

$$
w(x)=\frac{u(x)}{x^{q / p-1}} \exp \left(\frac{q}{p x} \int_{0}^{x} \ln \frac{1}{v(s)} d s\right) \quad \text { for } x \in(0, \infty) .
$$

Then the following statements are equivalent:

(i) There exists $C \in(0, \infty)$ such that the inequality

$$
\left(\int_{0}^{\infty}\left[\exp \left(\frac{1}{x} \int_{0}^{x} \ln f(t) d t\right)\right]^{q} u(x) d x\right)^{1 / q} \leq C\left(\int_{0}^{\infty} f^{p}(x) v(x) d x\right)^{1 / p}
$$

holds for all $f \in L_{+}^{p}((0, \infty) ; v)$.

(ii) There is $\lambda \in(0, \infty)$ such that the differential equation

$$
\lambda\left[\exp \left(\frac{q}{p} y^{\prime}(x)\right)\right]^{\prime}+\frac{w(x)}{x} \exp \left(\frac{q}{p} \frac{y(x)}{x}\right)=0
$$

has a solution $y$ satisfying the conditions

$$
y^{\prime} \in A C((0, \infty)), \quad \limsup _{x \rightarrow 0_{+}} y(x) \geq 0,
$$


and

$$
\int_{0}^{x} y^{\prime}(t) d t \text { exists for each } x>0 .
$$

(iii) The number $K$, defined by

$$
K:=\inf _{f \in P(0, \infty)} \sup _{x>0} \frac{1}{x f(x)} \int_{0}^{x} w(t) \exp f(t) d t
$$

is finite.

Moreover, if $C$ is the least constant for which (1.1) holds, then

$$
\sup _{\alpha>1}\left(\frac{\alpha-1}{\alpha}\right)^{1 / p} B(\alpha, q, p) \leq C \leq K^{1 / q} \leq e^{1 / q} B\left(1+\frac{p}{q}, q, p\right)
$$

where $B(\alpha, q, p)$ is given by (1.2), i.e.,

$$
B(\alpha, q, p)=\sup _{x>0} x^{(\alpha-1) / p}\left(\int_{x}^{\infty} \frac{w(t)}{t^{(\alpha-1) q / p+1}} d t\right)^{1 / q} .
$$

Proof of Theorem 2.1. Let the assumption of Theorem 2.1 be fulfilled.

The implication (iii) $\Rightarrow$ (ii) follows from

Lemma 2.2. Suppose $K<\infty$. Then, for each $\lambda>K$, the differential equation (2.2) has a solution $y$ satisfying (2.3) and (2.4).

Proof. Fix $\lambda>K$. According to (2.5) there exists $f \in P(0, \infty)$ such that, for all $x \in(0, \infty)$,

$$
f(x)>\frac{1}{\lambda x} \int_{0}^{x} w(t) \exp f(t) d t .
$$

Define a sequence of functions $z_{n}(x), x \in(0, \infty)$, by the formulas

$$
z_{0}(x)=f(x) \text {, }
$$

$$
z_{n}(x)=\frac{1}{\lambda x} \int_{0}^{x} w(t) \exp z_{n-1}(t) d t, \quad n \in \mathbb{N} .
$$

Obviously, $z_{n}(x)>0$ for $x>0$, and in view of (2.8) and (2.9) we have

$$
\int_{0}^{x} w(t) \exp z_{0}(t) d t<\infty \text { for all } x>0 .
$$

Moreover, according to (2.8) we have

$$
z_{0}(x)-z_{1}(x)=f(x)-\frac{1}{\lambda x} \int_{0}^{x} w(t) \exp f(t) d t>0
$$

and, consequently,

$$
z_{n}(x)-z_{n+1}(x)=\frac{1}{\lambda x} \int_{0}^{x} w(t)\left[\exp z_{n-1}(t)-\exp z_{n}(t)\right] d t>0 .
$$

Thus, the sequence $\left\{z_{n}\right\}$ is decreasing on $(0, \infty)$, which together with the positivity of $z_{n}$ yields the existence of a nonnegative function $z$ on $(0, \infty)$,

$$
z(x)=\lim _{n \rightarrow \infty} z_{n}(x) .
$$


Taking into account (2.11) and applying the monotone convergence theorem, we obtain from (2.10) that

$$
z(x)=\frac{1}{\lambda x} \int_{0}^{x} w(t) \exp z(t) d t, \quad x \in(0, \infty) .
$$

This formula implies that $z \in P(0, \infty) \cap A C((0, \infty))$ and $z$ satisfies the differential equation

$$
\lambda[x z(x)]^{\prime}=w(x) \exp z(x), \quad x \in(0, \infty) .
$$

For $c>0$ define

$$
y(x)=\frac{p x}{q} \int_{x}^{c} \frac{z(t)}{t} d t, \quad x>0
$$

Then

$$
y^{\prime}(x)=\frac{p}{q}\left[\int_{x}^{c} \frac{z(t)}{t} d t-z(x)\right],
$$

which implies the first condition in (2.3). Further, by (2.14), $y(x)>0$ for $x>0$ small enough, and consequently the second condition in (2.3) is also satisfied. Moreover, by (2.15),

$$
z(x)=\frac{q}{p}\left[\frac{y(x)}{x}-y^{\prime}(x)\right]
$$

and, hence,

$$
[x z(x)]^{\prime}=-\frac{q}{p} x y^{\prime \prime}(x) .
$$

Using this identity and (2.13) we obtain that $y^{\prime \prime}(x)<0$, and thus $y^{\prime}(x)$ is decreasing on $(0, \infty)$. Consequently, $\int_{0}^{x} y^{\prime}(t) d t$ exists for each $x>0$, so (2.4) is true. Finally, (2.17), (2.16), and (2.13) imply that $y$ is a solution of (2.2) and the lemma is proved.

The following lemma yields (ii) $\Rightarrow$ (i).

Lemma 2.3. Suppose that there is $\lambda \in(0, \infty)$ such that the differential equation (2.2) has a solution $y$ satisfying conditions (2.3) and (2.4). Then, for all $f \in$ $L_{+}^{p}((0, \infty) ; v)$,

$$
\left(\int_{0}^{\infty}\left[\exp \left(\frac{1}{x} \int_{0}^{x} \ln f(t) d t\right)\right]^{q} u(x) d x\right)^{1 / q} \leq \lambda^{1 / q}\left(\int_{0}^{\infty} f^{p}(x) v(x) d x\right)^{1 / p}
$$

Proof. Let $y$ be the solution of (2.2) satisfying (2.3) and (2.4). Then we have

$$
y(x) \geq y(x)-\limsup _{t \rightarrow 0_{+}} y(t)=\int_{0}^{x} y^{\prime}(t) d t .
$$

Suppose that $f \in L_{+}^{p}((0, \infty) ; v)$, and, for $x, t \in(0, \infty)$, denote

$$
\begin{gathered}
\phi(x)=-\lambda\left[\exp \left(\frac{q}{p} y^{\prime}(x)\right)\right]^{\prime}, \\
g(t)=f^{p}(t) v(t) \exp \left(-y^{\prime}(t)\right)
\end{gathered}
$$


Then (2.1), (2.21), Jensen's inequality, (2.19), (2.2), and (2.20) imply

$$
\begin{aligned}
{[\exp } & \left.\left(\frac{1}{x} \int_{0}^{x} \ln f(t) d t\right)\right]^{q} u(x) \\
= & \left\{\exp \left(\frac{1}{x} \int_{0}^{x} \ln \left[\frac{g(t)}{v(t)} \exp y^{\prime}(t)\right]^{1 / p} d t\right)\right\}^{q} \\
& \times w(x) x^{q / p-1} \exp \left(\frac{q}{p x} \int_{0}^{x} \ln v(t) d t\right) \\
= & w(x) x^{q / p-1}\left[\exp \left(\frac{1}{x} \int_{0}^{x} \ln g(t) d t\right)\right]^{q / p} \exp \left(\frac{q}{p x} \int_{0}^{x} y^{\prime}(t) d t\right) \\
\leq & w(x) x^{q / p-1}\left[\frac{1}{x} \int_{0}^{x} g(t) d t\right]^{q / p} \exp \left(\frac{q}{p x} y(x)\right) \\
\leq & \frac{w(x)}{x} \exp \left(\frac{q}{p} \frac{y(x)}{x}\right)\left(\int_{0}^{x} g(t) d t\right)^{q / p}=\phi(x)\left(\int_{0}^{x} g(t) d t\right)^{q / p} .
\end{aligned}
$$

Consequently,

$$
\begin{gathered}
\left(\int_{0}^{\infty}\left[\exp \left(\frac{1}{x} \int_{0}^{x} \ln f(t) d t\right)\right]^{q} u(x) d x\right)^{p / q} \\
\leq\left(\int_{0}^{\infty} \phi(x)\left(\int_{0}^{x} g(t) d t\right)^{q / p} d x\right)^{p / q}
\end{gathered}
$$

and the Minkowski integral inequality yields

$$
\left(\int_{0}^{\infty}\left[\exp \left(\frac{1}{x} \int_{0}^{x} \ln f(t) d t\right)\right]^{q} u(x) d x\right)^{p / q} \leq \int_{0}^{\infty} g(t)\left(\int_{t}^{\infty} \phi(x) d x\right)^{p / q} d t
$$

Since, according to (2.20),

$$
\left(\int_{t}^{\infty} \phi(x) d x\right)^{p / q} \leq \lambda^{p / q} \exp y^{\prime}(t)
$$

inequality (2.22) together with (2.21) implies (2.18).

The implication (i) $\Rightarrow$ (iii) is a consequence of (2.6), which follows from the next two assertions. The proof of Lemma 2.4 relies on the method used in [3].

Lemma 2.4. Suppose $p \in(0, \infty), q \in(0, \infty)$. Let there exist $C \in(0, \infty)$ such that $(1.1)$ holds for all $f \in L_{+}^{p}((0, \infty) ; v)$. Then, for arbitrary $\alpha \in(1, \infty)$,

$$
B(\alpha, q, p) \leq\left(\frac{\alpha}{\alpha-1}\right)^{1 / p} C \text {. }
$$

Proof. Let $\alpha \in(1, \infty)$. Fix $t>0$ and put

$$
f(s)=[g(s) / v(s)]^{1 / p},
$$

where

$$
g(s)=t^{-\beta} \chi_{(0, t)}(s)+t^{\alpha-\beta} s^{-\alpha} \chi_{(t, \infty)}(s), \quad s>0
$$


and $\beta \in \mathbb{R}\left(\chi_{E}\right.$ denotes the characteristic function of $\left.E\right)$. Then

$$
\left(\int_{0}^{\infty} f^{p}(x) v(x) d x\right)^{1 / p}=\left(\frac{\alpha}{\alpha-1}\right)^{1 / p} t^{(1-\beta) / p}
$$

In view of (2.24) we have

$$
\begin{aligned}
& \left(\int_{0}^{\infty}\left[\exp \left(\frac{1}{x} \int_{0}^{x} \ln f(s) d s\right)\right]^{q} u(x) d x\right)^{1 / q} \\
& \quad=\left(\int_{0}^{\infty}[G(x)]^{q / p} z(x) d x\right)^{1 / q} \geq\left(\int_{t}^{\infty}[G(x)]^{q / p} z(x) d x\right)^{1 / q},
\end{aligned}
$$

where

$$
\begin{gathered}
G(x)=\exp \left(\frac{1}{x} \int_{0}^{x} \ln g(s) d s\right) \\
z(x)=\left[\exp \left(\frac{1}{x} \int_{0}^{x} \ln \frac{1}{v(s)} d s\right)\right]^{q / p} u(x) .
\end{gathered}
$$

If $x>t$, then, by (2.25) and (2.28),

$$
G(x)=t^{\alpha-\beta}(e / x)^{\alpha} \exp (-\alpha t / x),
$$

and, since $\exp (-\alpha t / x)>e^{-\alpha}$, we have

$$
G(x)>t^{\alpha-\beta} x^{-\alpha} \text { for } x>t .
$$

This estimate and (2.27) imply

$$
\left(\int_{0}^{\infty}\left[\exp \left(\frac{1}{x} \int_{0}^{x} \ln f(s) d s\right)\right]^{q} u(x) d x\right)^{1 / q} \geq t^{(\alpha-\beta) / p}\left\{\int_{t}^{\infty} \frac{z(x)}{x^{\alpha q / p}} d x\right\}^{1 / q}
$$

Using (2.26) and (2.29) in (1.1), we obtain

$$
t^{(\alpha-\beta) / p}\left(\int_{t}^{\infty} \frac{z(x)}{x^{\alpha q / p}} d x\right)^{1 / q} \leq\left(\frac{\alpha}{\alpha-1}\right)^{1 / p} t^{(1-\beta) / p} C,
$$

and, hence, since $t$ was arbitrary, (2.23) follows.

Lemma 2.5. Let $C$ be the least possible constant in (1.1), let $K$ be defined by (2.5), and let $B(\alpha, q, p)$ be given by (2.7). Then

$$
C \leq K^{1 / q} \leq e^{1 / q} B\left(1+\frac{p}{q}, q, p\right) \text {. }
$$

Proof. (a) We shall prove the first inequality in (2.30) by contradiction. To this end, let us assume that $K^{1 / q}<C$, and choose $\lambda_{0}$ such that

$$
K^{1 / q}<\lambda_{0}^{1 / q}<C \text {. }
$$

Since $K<\lambda_{0}$, the problem (2.2)-(2.4) is solvable for $\lambda=\lambda_{0}$ due to Lemma 2.2. Moreover, $C \leq \lambda_{0}^{1 / q}$ by Lemma 2.3 , which contradicts (2.31).

(b) If $B:=B\left(1+\frac{p}{q}, q, p\right)=\infty$, then the second inequality in (2.30) holds trivially. Therefore, assume $B<\infty$. According to (2.7),

$$
B^{q}=\sup _{x>0} x W(x), \quad \text { where } W(x)=\int_{x}^{\infty} \frac{w(t)}{t^{2}} d t
$$


Thus,

$$
x W(x) \leq B^{q}<e B^{q} \text { for all } x>0 .
$$

Consequently, putting

$$
f_{0}(x)=\ln \frac{e B^{q}}{x W(x)}, \quad x>0,
$$

we have $f_{0} \in P(0, \infty)$, and hence

$$
K \leq \sup _{x>0} \frac{1}{x f_{0}(x)} \int_{0}^{x} w(t) \exp f_{0}(t) d t=e B^{q} \sup _{x>0} I(x)\left(x f_{0}(x)\right)^{-1},
$$

where

$$
I(x)=\int_{0}^{x} t \frac{w(t)}{t^{2}} \frac{d t}{W(t)} \text { for } x>0 .
$$

Integrating by parts and making use of (2.32), we obtain

$$
\begin{aligned}
I(x) & \leq-x \ln W(x)+\int_{0}^{x} \ln W(t) d t \\
& \leq-x \ln W(x)+x+x \ln \left(B^{q} / x\right)=x f_{0}(x) .
\end{aligned}
$$

This estimate together with (2.33) implies $K^{1 / q} \leq e^{1 / q} B$, and the proof is complete.

Example 2.6. Suppose $p \in(0, \infty), q \in[p, \infty)$.

(i) Let $v \in \mathscr{W}(0, \infty)$ be such that

$$
\int_{0}^{x}|\ln v(s)| d s<\infty \text { for each } x>0 .
$$

Setting

$$
u(x)=x^{q / p-1}\left[\exp \left(\frac{1}{x} \int_{0}^{x} \ln v(t) d t\right)\right]^{q / p} \text { for } x>0,
$$

we easily verify that $B(\alpha, q, p)=(\alpha-1)^{-1 / q}(p / q)^{1 / q}$ for arbitrary $\alpha>1$. Hence we have

$$
B\left(1+\frac{p}{q}, q, p\right)=1 \text {. }
$$

Thus, by Theorem 2.1,

$$
\begin{aligned}
& \left(\int_{0}^{\infty}\left[\exp \left(\frac{1}{x} \int_{0}^{x} \ln f(t) d t\right)\right]^{q} u(x) d x\right)^{1 / q} \\
& \leq e^{1 / q}\left(\int_{0}^{\infty} f^{p}(x) v(x) d x\right)^{1 / p}
\end{aligned}
$$

for all $f \in L_{+}^{p}((0, \infty) ; v)$. In particular, choosing $v(x) \equiv 1$, we obtain that the inequality

$$
\begin{aligned}
& \left(\int_{0}^{\infty}\left[\exp \left(\frac{1}{x} \int_{0}^{x} \ln f(t) d t\right)\right]^{q} x^{q / p-1} d x\right)^{1 / q} \\
& \quad \leq e^{1 / q}\left(\int_{0}^{\infty} f^{p}(x) d x\right)^{1 / p}
\end{aligned}
$$


holds for every $f \in L_{+}^{p}((0, \infty))$. If, in addition, $q=p=1$, then the inequality (2.35) is that of Knopp (cf. [2, Theorem 335]).

Taking

$$
v(s)=e^{g(s)} \quad \text { for } s>0,
$$

where

$$
\int_{0}^{x}|g(s)| d s<\infty \text { for all } x>0,
$$

inequality (2.34) turns to

$$
\begin{aligned}
& \left(\int_{0}^{\infty}\left[\exp \left(\frac{1}{x} \int_{0}^{x} \ln f(t) d t\right)\right]^{q} \exp \left(\frac{q}{p x} \int_{0}^{x} g(s) d s\right) x^{q / p-1} d x\right)^{1 / q} \\
& \quad \leq e^{1 / q}\left(\int_{0}^{\infty} f^{p}(x) e^{g(x)} d x\right)^{1 / p} .
\end{aligned}
$$

For example, if $g(s)=n \beta s^{n-1}$ for $s>0, n \in \mathbb{N}, \beta \in \mathbb{R}$, we have that all $f \in L_{+}^{p}\left((0, \infty) ; \exp \left(n \beta x^{n-1}\right)\right)$ satisfy

$$
\begin{aligned}
& \left(\int_{0}^{\infty}\left[\exp \left(\frac{1}{x} \int_{0}^{x} \ln f(t) d t\right)\right]^{q} \exp \left(\frac{q}{p} \beta x^{n-1}\right) x^{q / p-1} d x\right)^{1 / q} \\
& \leq e^{1 / q}\left(\int_{0}^{\infty} f^{p}(x) \exp \left(n \beta x^{n-1}\right) d x\right)^{1 / p} .
\end{aligned}
$$

(ii) Let $u \in \mathscr{W}(0, \infty)$ be such that

$$
x \ln \left(u(x) x^{1-q / p}\right) \in A C((0, \infty))
$$

and

$$
\lim _{x \rightarrow 0_{+}} x \ln \left(u(x) x^{1-q / p}\right) \leq 0
$$

Taking

$$
v(x)=\exp \left(\frac{d}{d x}\left[\frac{p x}{q} \ln \left(u(x) x^{1-q / p}\right)\right]\right) \text { for } x>0,
$$

we have $B(\alpha, q, p) \leq(\alpha-1)^{-1 / q}(p / q)^{1 / q}$ for arbitrary $\alpha>1$ and, consequently,

$$
B\left(1+\frac{p}{q}, q, p\right) \leq 1 \text {. }
$$

Thus, by Theorem $2.1,(2.34)$ holds for all $f \in L_{+}^{p}((0, \infty) ; v)$.

\section{REFERENCES}

1. J. A. Cochran and C.-S. Lee, Inequalities related to Hardy's and Heinig's, Math. Proc. Cambridge Philos. Soc. 96 (1984), 1-7.

2. G. H. Hardy, J. E. Littlewood, and G. Pólya, Inequalities, Cambridge Univ. Press, 1964.

3. H. P. Heinig, Weighted inequalities in Fourier analysis, Nonlinear Analysis, Function Spaces and Applications, Vol. 4, Teubner-Texte Math., band 119, Teubner, Leipzig, 1990, pp. 42-85.

4. E. R. Love, Inequalities related to those of Hardy and Cochran and Lee, Math. Proc. Cambridge Philos. Soc. 99 (1986), 395-408.

5. G. Talenti, Una diseguaglianza integrale, Boll. Un. Mat. Ital. (3) 21 (1966), 25-34. 
6. __ Sopra una diseguaglianza integrale, Ann. Scuola Norm. Sup. Pisa (3) 21 (1967), 167-188.

7. G. Tomaselli, A class of inequalities, Boll. Un. Mat. Ital. (4) 2 (1969), 622-631.

Mathematical Institute, Czech Academy of Sciences, Žitná 25, 11567 Prague 1, Czech REPUBLIC

E-mail address: opic@csearn.bitnet

Department of Mathematics, University of Agriculture, 16521 Prague 6, Czech RePUBLIC

E-mail address: gurka@csearn.bitnet 